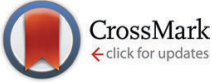

Cite this: Phys. Chem. Chem. Phys., 2016, 18, 32891

Received 15th August 2016 Accepted 1st November 2016

DOI: $10.1039 / c 6 c p 05661$

www.rsc.org/pccp

\title{
Surface induced vibrational modes in the fluorescence spectra of PTCDA adsorbed on the $\mathrm{KCl}(100)$ and $\mathrm{NaCl}(100)$ surfaces
}

\author{
A. Paulheim, ${ }^{a}$ C. Marquardt, ${ }^{a}$ M. Sokolowski, ${ }^{\star a}$ M. Hochheim, ${ }^{b}$ T. Bredow, ${ }^{b}$ \\ H. Aldahhak, ${ }^{C}$ E. Rauls ${ }^{c}$ and W. G. Schmidt ${ }^{c}$
}

\begin{abstract}
We report a combined experiment-theory study on low energy vibrational modes in fluorescence spectra of perylene-3,4,9,10-tetracarboxylic acid dianhydride (PTCDA) molecules. Using very low coverages, isolated molecules were adsorbed on terrace sites or at sites located at residual steps on (100) oriented alkali halide films $(\mathrm{KCl}$ and $\mathrm{NaCl}$ ). The low energy modes couple to the optical transition only because the PTCDA molecule is geometrically distorted $\left(C_{2 v}\right)$ upon adsorption on the surface; they would be absent for the parent planar $\left(D_{2 h}\right)$ PTCDA molecule. The modes differ in number and energy for molecules adsorbed on regular terrace sites and molecules adsorbed at step edge sites. Modes appearing for step edge sites have the character of frustrated rotations. Their coupling to the optical transition is a consequence of the further reduced symmetry of the step edge sites. We find a larger number of vibrational modes on $\mathrm{NaCl}$ than on $\mathrm{KCl}$. We explain this by the stronger electrostatic bonding of the PTCDA on $\mathrm{NaCl}$ compared to $\mathrm{KCl}$. It causes the optical transition to induce stronger changes in the molecular coordinates, thus leading to larger Franck-Condon factors and thus stronger coupling. Our results demonstrate how optical spectroscopy can be used to gain information on adsorption sites of molecules at low surface concentrations.
\end{abstract}

\section{Introduction}

\subsection{Motivation}

The spectroscopy of the vibrational modes of molecules adsorbed on surfaces is a versatile experimental tool for obtaining information about the geometric structure of the adsorption complex. In particular, vibrational modes at low energies which are subject to coupling to surface atoms are interesting for this purpose. So far, mainly high resolution energy electron loss spectroscopy (HREELS) has been used to measure these modes. ${ }^{1}$ In the present work we demonstrate that fluorescence (FL) spectroscopy also gives access to low energy vibrational modes of adsorbates, which, in addition to a high signal-to-noise ratio, ${ }^{2}$ bears the possibility to distinguish different adsorption sites by their optical transition energies. We support our experimental data with theoretical calculations showing in detail, how the structure and symmetry of the adsorption sites influence the vibrational modes in the fluorescence spectra.

\footnotetext{
${ }^{a}$ Institut für Physikalische und Theoretische Chemie der Universität Bonn, Wegelerstraße 12, 53115 Bonn, Germany.E-mail: sokolowski@pc.uni-bonn.de

${ }^{b}$ Mulliken Center for Theoretical Chemistry, Institut für Physikalische und Theoretische Chemie der Universität Bonn, Beringstraße 4, 53115 Bonn, Germany ${ }^{c}$ Lehrstuhl für Theoretische Physik, Universität Paderborn, Warburger Straße 100, 33098 Paderborn, Germany
}

We report FL spectra of very high resolution of perylene3,4,9,10-tetracarboxylic acid dianhydride (PTCDA, see Fig. 1) molecules adsorbed on thin (100) oriented films of $\mathrm{KCl}$ and $\mathrm{NaCl}$. PTCDA molecules adsorbed on these surfaces constitute an interesting model system for the adsorption of larger $\pi$-conjugated molecules on insulator surfaces. This topic has gained attention over the last years, ${ }^{3-6}$ because there are interesting differences from the adsorption of these molecules on metal surfaces. ${ }^{7}$ For instance, on insulator surfaces, the bonding is dominated by electrostatic and van der Waals interactions. Covalent bond formation, which is often observed on metal surfaces, ${ }^{7}$ is less dominant on insulators and molecular properties are thus often better accessible than for molecules adsorbed on metallic surfaces. Nevertheless, the surface/adsorbate interactions on insulator surfaces can also be strong and site specific. For instance, for PTCDA on the $\mathrm{KCl}(100)$ surface, the Coulomb interactions between partial charges on the carboxylic oxygen atoms and the surface cations lead to adsorption on specific surface sites and formation of commensurate superstructures. ${ }^{8-10}$

The planar PTCDA molecule belongs to the symmetry group $D_{2 \mathrm{~h}}$ and exhibits 108 vibrational modes in total; ${ }^{11} 46$ of these are infrared active ( $\sim$ representations $\left.B_{1 u}, B_{2 u}, B_{3 u}\right), 54$ are Raman active ( $\sim$ representations $\left.\mathrm{A}_{\mathrm{g}}, \mathrm{B}_{1 \mathrm{~g}}, \mathrm{~B}_{2 \mathrm{~g}}, \mathrm{~B}_{3 \mathrm{~g}}\right)$, and 8 modes are silent $\left(\sim\right.$ representation $\left.A_{u}\right){ }^{12}$ In this work the optical $S_{0} / S_{1}$ 

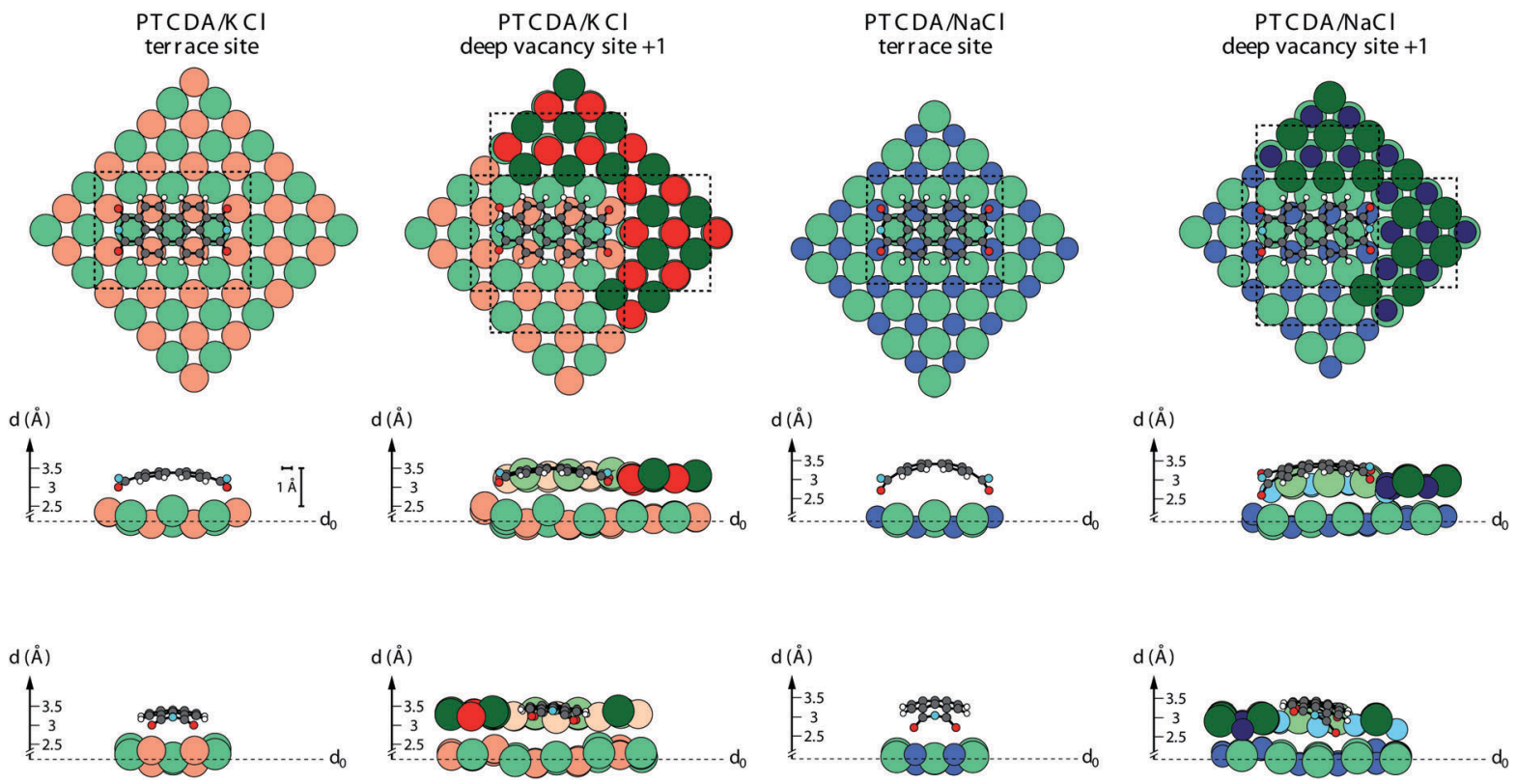

Fig. 1 Top: Hardsphere models (top view) of the adsorption geometry of PTCDA on terrace sites (t sites) and at step edge sites (s site, here: the so called deep vacancy site +1$)$ on $\mathrm{KCl}(100)$ and $\mathrm{NaCl}(100)$. All atomic and ionic positions are from DFT calculations. The black dashed rectangles in the top views indicate which ions can also be seen in the respective side views. For the adsorption at the step edges different types of sites exist which differ slightly by additional rows of ions. These sites are described and discussed in detail in ref. 18. The site shown here as an example is considered to be the most typical one and is called "deep vacancy site +1 ". Middle/bottom: Side views along the short (middle) and along the long molecular axis of the PTCDA molecule in the direction towards the step (bottom). H atoms are shown in white, carbon atoms in gray, carboxylic and anhydride oxygen atoms in red/cyan, sodium/ potassium ions in blue/red, and chlorine in green. Heavier and lighter shades of blue/red and green are used to illustrate the step edge geometry. In the side views, $d$ denotes the vertical adsorption height; the vertical scale is enlarged by a factor of 4 for all atoms and ions in order to emphasize the vertical distortions of the molecules and the buckling of the substrate. $d_{0}$ indicates the vertical height of the $\mathrm{Cl}^{-}$ion below the center of the perylene core.

transition of PTCDA was investigated. For the planar molecule, only the 19 in-plane $A_{g}$ modes couple to the $\mathrm{S}_{0} / \mathrm{S}_{1}$ transition. ${ }^{13}$ The energetically lowest $A_{g}$ mode is at about $232 \mathrm{~cm}^{-1} .{ }^{13}$ Thus, all vibrational modes at lower energies which we describe in this work are special. They are out-of-plane modes and do not belong to the $\mathrm{A}_{\mathrm{g}}$ representation of the undistorted and planar PTCDA molecule. They couple to the optical transition only, because the symmetry of the adsorption complex on the $\mathrm{KCl}$ and $\mathrm{NaCl}$ surfaces is lower than that of the parent molecule, namely $C_{2 \mathrm{v}}$ (see below). In this case, modes of the $\mathrm{B}_{3 \mathrm{u}}$ representation of the planar molecule with $D_{2 \mathrm{~h}}$ symmetry, i.e. modes which include out-of-plane components, project into the totally symmetric representation $\mathrm{A}_{1}$ of $C_{2 \mathrm{v}}$ and thus couple to the optical transition. The aspect that these low energy vibrational modes do not couple to the $S_{0} / S_{1}$ transition of the planar molecule was experimentally confirmed by fluorescence spectra of PTCDA in He nanodroplets. ${ }^{14-16}$

For a strongly allowed transition, as it is considered here, the coupling to vibrational modes is described by the respective Franck-Condon (FC) factors. ${ }^{17}$ Accordingly, the intensity of the first fundamental vibrational mode (frequency $\omega_{i}$, reduced mass $M_{i}$ ) is proportional to the Huang-Rhys factor $S_{i}=\left(M_{i} \omega_{i} / 2 \hbar\right) \times \Delta q_{i}^{2}$, where $\Delta q_{i}$ denotes the displacement of the minimum of the potential along the respective normal coordinate $q_{i}$ under the transition. Obviously, only modes with $\Delta q_{i} \neq 0$, i.e. those for which the equilibrium distance changes, couple to the transition. For the low energy modes considered here, this implies that the molecule changes its out-of-plane distortion during the optical transition. Strictly speaking, the ground or the excited state has to be distorted, but, of course, it is reasonable to assume a distortion of both. The observation of low energy vibrational modes in the optical spectrum is thus a fingerprint for an outof-plane distortion of PTCDA as a consequence of the surface bonding. From the Huang-Rhys factors one can also gain an intuitive understanding of the coupling strength which is helpful: if the charge displacement due to the optical transition modifies the Coulomb interactions between the atoms of the molecule and the ions of the surface, displacements of normal coordinates can be expected and will lead to coupling of the respective modes to the optical transition. Importantly, here the respective normal coordinates refer to both the intramolecular distances and the adsorption geometry of the molecule with respect to the surface.

In the following, we name these modes which appear only in the optical spectrum for PTCDA adsorbed on a surface "surface induced modes" (SIM). As we report, these modes differ for the two studied surfaces, $\mathrm{KCl}(100)$ and $\mathrm{NaCl}(100)$, albeit the adsorption sites are principally identical. We will interpret this difference by the stronger bonding of PTCDA on NaCl compared to $\mathrm{KCl}$. In addition, we find different modes for molecules adsorbed on terraces and at step edge sites, revealing the presence of different symmetries on these two types of adsorption sites. The appearance of SIM on $\mathrm{KCl}$ was briefly reported by us in ref. 19 . In the present work, we give a more detailed analysis on the basis of spectra measured with higher resolution. In addition, we here 
include data on the SIM for PTCDA on the $\mathrm{NaCl}(100)$ surface and compare these with those on the $\operatorname{KCl}(100)$ surface.

\subsection{Background information}

Information on the adsorption sites of PTCDA on $\mathrm{KCl}$ and $\mathrm{NaCl}(100)$ has recently been gained from STM data ${ }^{20,21}$ and density functional theory (DFT) calculations. ${ }^{18,20,22,23}$ From DFT calculations it was found that in the favored adsorption complex the center of the perylene core is on top of $\mathrm{a} \mathrm{Cl}^{-}$anion. The four carboxylic oxygen atoms $\left(\mathrm{O}_{\text {carb }}\right)$ are close to surface cations, "anchoring" the molecule on its four corners to the surface by attractive Coulomb interactions. ${ }^{22,24}$ The long molecular axis is oriented in the [011] direction of the substrate. These adsorption configurations are illustrated in Fig. 1. The bonding of the $\mathrm{O}_{\text {carb }}$ atoms to the surface causes the PTCDA molecules to undergo an arch-type distortion. The resulting symmetry of the adsorption complex is $C_{2 \mathrm{v}}$. The center of the perylene core is $3.40 \AA$ above the $\mathrm{Cl}^{-}$anions on both surfaces. ${ }^{22}$ The four carboxylic $\mathrm{O}$ atoms are shifted vertically downward from this height by $0.4 \AA$ and $0.7 \AA$ on $\mathrm{KCl}$ and $\mathrm{NaCl}$, respectively. The significantly stronger distortion of PTCDA on $\mathrm{NaCl}$ compared to $\mathrm{KCl},{ }^{22,23}$ proposed from the DFT calculations, can be understood as a result of the smaller lattice constant of $\mathrm{NaCl}$ (5.640 A (ref. 25)) compared to that of $\mathrm{KCl}$ (6.293 $\AA$ (ref. 25)). This leads to a better fit of the negatively charged $\mathrm{O}_{\text {carb }}$ atoms on the four corners of the molecule to the underlying surface cations ${ }^{26,27}$ (see Fig. 1). As a result, the Coulombic attractions of the $\mathrm{O}_{\text {carb }}$ atoms to the $\mathrm{NaCl}$ surface are larger and cause a stronger out-ofplane distortion than on $\mathrm{KCl}$. We note that, differently from the situation on metal surfaces, ${ }^{7}$ quantitative experimental determinations of the bonding heights and the distortions of PTCDA on the $\mathrm{KCl}$ or $\mathrm{NaCl}(100)$ surface are missing.

The optical properties of isolated PTCDA molecules adsorbed on $\mathrm{KCl}(100)$ films ${ }^{19,28}$ and $\mathrm{NaCl}(100)$ films ${ }^{2,29}$ have been reported already. Important in the context of the present work is the aspect that one can distinguish the population of different adsorption sites from in the optical spectra. Directly after deposition onto the cold sample, the PTCDA molecules populate predominantly terraces sites ( $\mathrm{t}$ sites). Thermal annealing or exposure to intense optical radiation promote diffusion of the PTCDA to sites located at step edges of the substrate (s sites). ${ }^{28}$ For the considered low coverages of molecules the small number of steps from growth defects on the nominally flat films is sufficient for providing step sites for all molecules. The azimuthal orientation of the molecules is identical on $\mathrm{s}$ and $\mathrm{t}$ sites. $^{28}$ The adsorption at the $s$ sites is however thermodynamically more stable and the process is irreversible. From recent STM data and DFT calculations, the s sites on $\mathrm{KCl}$ were identified as so-called vacancy sites, because they stabilize the PTCDA adsorption by partially embedding the molecule into the step edge. ${ }^{20}$ A similar situation exists on the $\mathrm{NaCl}(100)$ surface, and was derived from analogous $\mathrm{STM}^{21}$ and DFT data. ${ }^{18,22}$ Importantly, small variations in the local arrangement of the ions around the PTCDA are possible; the details are reported in ref. 16. The $t$ sites and one of the most typical $s$ sites are illustrated in Fig. 1. On the $\mathrm{KCl}(100)$ surface, a concomitant blue shift of the
$\mathrm{S}_{0} / \mathrm{S}_{1}$ transition by $130 \pm 15 \mathrm{~cm}^{-1}$ was observed for the transition of the molecules from $\mathrm{t}$ to $\mathrm{s}$ sites. ${ }^{28}$ For the $\mathrm{NaCl}$ surface, a very similar spectral shift is reported here.

\section{Experimental and computational details}

\subsection{Experimental details}

The PTCDA molecules were sublimated from a Knudsen cell onto thin epitaxial $\mathrm{KCl}(100)$ and $\mathrm{NaCl}(100)$ films under ultrahigh vacuum. The $\mathrm{KCl}$ and $\mathrm{NaCl}$ films of about 10 atomic layers in thickness were both grown on a single $\mathrm{Ag}(100)$ sample as described earlier in ref. 9 and 30, respectively. During the PTCDA deposition, the sample was kept between 6 and $20 \mathrm{~K}$ in all experiments. At these low temperatures the molecules stick to the surface in a planar and azimuthally defined orientation on defined adsorption sites on the terraces ( $\mathrm{t}$ sites); however, they are laterally immobile. ${ }^{19}$ For inducing the transition of the molecules to adsorption sites at steps (s sites) we heated the sample at $100-150 \mathrm{~K}$ for about 10 minutes. ${ }^{28}$ For the details of the PTCDA deposition and coverage calibration we refer to ref. 28. We used coverages between $0.02 \%$ and $1.00 \%$ of a monolayer, whereby one monolayer is equivalent to $7.5 \times 10^{-3}$ molecules per $\AA^{2}$.

For the optical experiments we transferred the sample into a glass tube standing out at the end of the UHV chamber. For FL spectroscopy we used an $\mathrm{Ar}^{+}$laser (Coherent Innova Sabre ${ }^{\mathrm{TM}}$ Ion Laser) and a solid state diode pumped laser (Sapphire LP USB $\mathrm{CDRH})$ operated at $457.9 \mathrm{~nm}$ and $458 \mathrm{~nm}\left(21834 \mathrm{~cm}^{-1}\right)$, respectively. For measuring the $\mathrm{t}$ site spectra the excitation intensities were reduced in order avoid light induced migration to $\mathrm{s}$ sites. ${ }^{28}$ The FL light was measured with a spectrometer (Acton 2300i, $f / \#=4, f=0.3 \mathrm{~m}$ ) equipped with a liquid nitrogen cooled CCD camera (Spec-10:100BR(LN)). The experimental resolution of the FL spectra is determined by the spectrometer and amounts to 5-9 $\mathrm{cm}^{-1}$ for a grating of 1200 grooves per $\mathrm{mm}$. The optical spectra were recorded at sample temperatures between 6 and $20 \mathrm{~K}$. We note that in the FL spectra, which we consider here, the transition couples to vibrational modes of the ground state $\left(\mathrm{S}_{0}\right)$, i.e., similar to other vibrational surface spectroscopies, e.g., high resolution energy electron loss spectroscopy (HREELS), ${ }^{1}$ contrary to optical absorption spectroscopy which couples to vibrational modes of the excited state $\left(\mathrm{S}_{1}\right)$.

\subsection{Computational details}

The DFT calculations underlying the structural data presented in Fig. 1 were described in detail in ref. 18, 20 and 22. For calculating the vibrational modes and the respective FC factors a second set of DFT calculations was performed on the GGAlevel of theory (PBED3 ${ }^{31-34}$ ) including dispersion corrections within the crystalline-orbital program CRYSTAL14. ${ }^{35}$ The full details of these calculations will be reported in ref. 36; here we summarize some of the most important points required for the understanding of our comparison of the calculations to the experimental data. Due to the use of the periodic CRYSTAL14 
code we excluded boundary effects and took surface relaxations into account. An extra fine integration grid (XXLGRID) and tight tolerances for two-electron integrals have been used. We note that these calculations gave the same geometries for the ground states as those performed earlier. ${ }^{18,20,22}$

Because the electronic excitation takes place in the molecule and the excited vibrations are primarily located on the molecule, we decided to use relatively small basis sets for the surface atoms sodium, ${ }^{37,38}$ potassium, ${ }^{37,38}$ and chlorine, ${ }^{39}$ and the larger $6-311 G^{* *}$ Pople-type basis of triple- $\zeta$-quality was used for the atoms of the PTCDA molecule. ${ }^{40}$ Because calculations of the optically excited state cannot be performed within CRYSTAL14, we used the quantum chemical program package Gaussian09 for the purpose of excited state and frequency calculations. ${ }^{41}$ All excited-state properties were calculated on the time-dependent DFT (TD-DFT) level of theory, applying the range-separated hybrid functional CAM-B3LYP. ${ }^{42,43}$ Dispersion forces were again added via the D3 dispersion correction. The same basis sets were used for the surface as it was done with CRYSTAL14, but we truncated the molecular basis set to $6-311 G^{*}$ to reduce computational costs. In Gaussian09 the adsorbate system was modeled with an embedded cluster approach, where the atoms of the surface cluster were embedded in point charges of $\pm 1.0 \mathrm{e}$. The surface atom positions of the adsorbate-cluster system were taken from our periodic calculations. For consistency reasons we re-optimized the geometry of PTCDA in the $\mathrm{S}_{0}$ and the $\mathrm{S}_{1}$ state and allowed free relaxation of the molecule, while the surface atoms were kept fixed. In order to model the molecule at the $t$ and at the s sites, two different types of clusters were used. For modeling the PTCDA adsorption at a t site a surface cluster of $C_{2 \mathrm{v}}$ symmetry was used. For correct calculations of the PTCDA on the s sites of $C_{1}$ symmetry the presence of the step atoms requires the use of structurally much more complicated and larger cluster models (see Fig. 1). At present such models are computationally too demanding. Therefore, we have limited ourselves to calculations on an adsorbate/cluster model which does not include the step atoms yet, but exhibits the deliberately reduced and correct symmetry, namely $C_{1}$, that is expected for the s sites. We used this model as a first order approximation for the molecules adsorbed at the s sites. It exhibits the correct symmetry, but obviously the detailed structural environment differs from that at the real $\mathrm{s}$ sites due to the absence of the step atoms. Detailed structure models of these clusters are given in ref. 36.

Subsequently, we calculated the vibrations of the molecule in the $S_{0}$ and the $S_{1}$ state. During this calculation the surface atoms were again kept at the fixed positions of the adsorbatecluster. The obtained geometries, vibrational frequencies and normal modes of the $S_{0}$ and the $S_{1}$ state were finally used to calculate the FC-factors with the program ezSpectrum ${ }^{44}$ applying the parallel approximation with previous normal mode reordering. The FC factors were normalized such that the value of the FC factor at the first vibrational in-plane mode $\nu_{1}$ corresponded to the intensity of this mode in the normalized FL spectrum, i.e., the spectrum where the $0-0$ intensity was set to 1 . Typically FC factors of the $\nu_{1}$ mode were about 0.3 . An intensity threshold of 0.001 was applied to all modes.

\section{Results and discussion}

\subsection{Surface induced vibrational modes}

Fig. 2 gives an overview on the FL spectra in the range of small vibrational energies (below $300 \mathrm{~cm}^{-1}$ ) which were measured directly after the deposition, when the molecules are mainly on t sites (upper spectra), and after additional thermal annealing, when the molecules have diffused to the s sites (lower spectra). For all these spectra the vibrational energies are referenced with respect to the pure electronic transitions, i.e. the $0-0$ transitions. The absolute energies of the respective $0-0$ transitions can be found in the figure caption. The blue shift of the 0-0 transition on $\mathrm{NaCl}$ upon migration of the molecules to the step edges amounts to $144 \mathrm{~cm}^{-1}$ and is thus very similar to the average blue shift of $135 \pm 15 \mathrm{~cm}^{-1}$ observed on $\mathrm{KCl} . \dagger$ For both systems the blue shifts of the transitions are related to the diffusion of the molecules to the sites and the resulting changes in the adsorption energies of the $S_{0}$ and $S_{1}$ states. ${ }^{45} \mathrm{We}$ also note that the line widths are strongly reduced by a factor of $\sim 5$ upon the migration of the molecules to the s sites due to smaller inhomogeneous line broadening. This aspect was described in detail in ref. 45. Since the inhomogeneous line broadening also affects the line shape of the vibrational modes, the s site spectra generally exhibit sharper and hence better to resolve vibrational modes.

In Fig. 2 we have compiled several spectra for different preparation routines in order to show the sample-to-sample variations (gray spectra). The averaged spectra are shown as the heavy lines. Notably, all spectra are given on a logarithmic scale in order to enhance the visibility of the small peaks. The positions of the peaks are summarized in Table 1. The strong vibrational peak at $231(228) \mathrm{cm}^{-1}$ on $\mathrm{NaCl}(\mathrm{KCl})$ is due to an in-plane breathing mode of the molecules and is also present in optical spectra of the planar molecules with $D_{2 \mathrm{~h}}$ symmetry. ${ }^{16}$ All other peaks at smaller vibrational energies are surface induced modes (SIM) that have been described in the introduction and which will be in the focus of this contribution.

Before we concentrate on the SIM, we comment on the vibrational modes at energies above $230 \mathrm{~cm}^{-1}$. For this purpose, we compare wide range FL spectra taken for the s sites on $\mathrm{KCl}$ and $\mathrm{NaCl}$ in Fig. 3. The respective vibrational energies of the peaks are given in Table 1. The important information from these wide range spectra is that the energetic positions of all lines above $230 \mathrm{~cm}^{-1}$, which correspond to fundamental vibrational in-plane modes of the PTCDA, are identical within $10 \mathrm{~cm}^{-1}$ on both surfaces. This is interesting in so far, as it indicates that the lateral in-plane modes are identical on both substrates. These vibrational energies also correspond well to those of the vibrational modes in the spectra measured for molecules on $t$ sites on $\mathrm{KCl}$ and $\mathrm{NaCl}$ (see Table 1). For a detailed interpretation of these modes we refer to Scholz et al. ${ }^{13}$ We note that the vibrational energies for the $\mathrm{KCl}$ t sites in Table 1 are based on more recent and better resolved spectra and thus partially differ

$\dagger$ This is the average value calculated from the spectra included in Fig. 2. The value is about $4 \%$ larger than the one reported earlier ${ }^{18,27}$ based on a different set of spectra. 

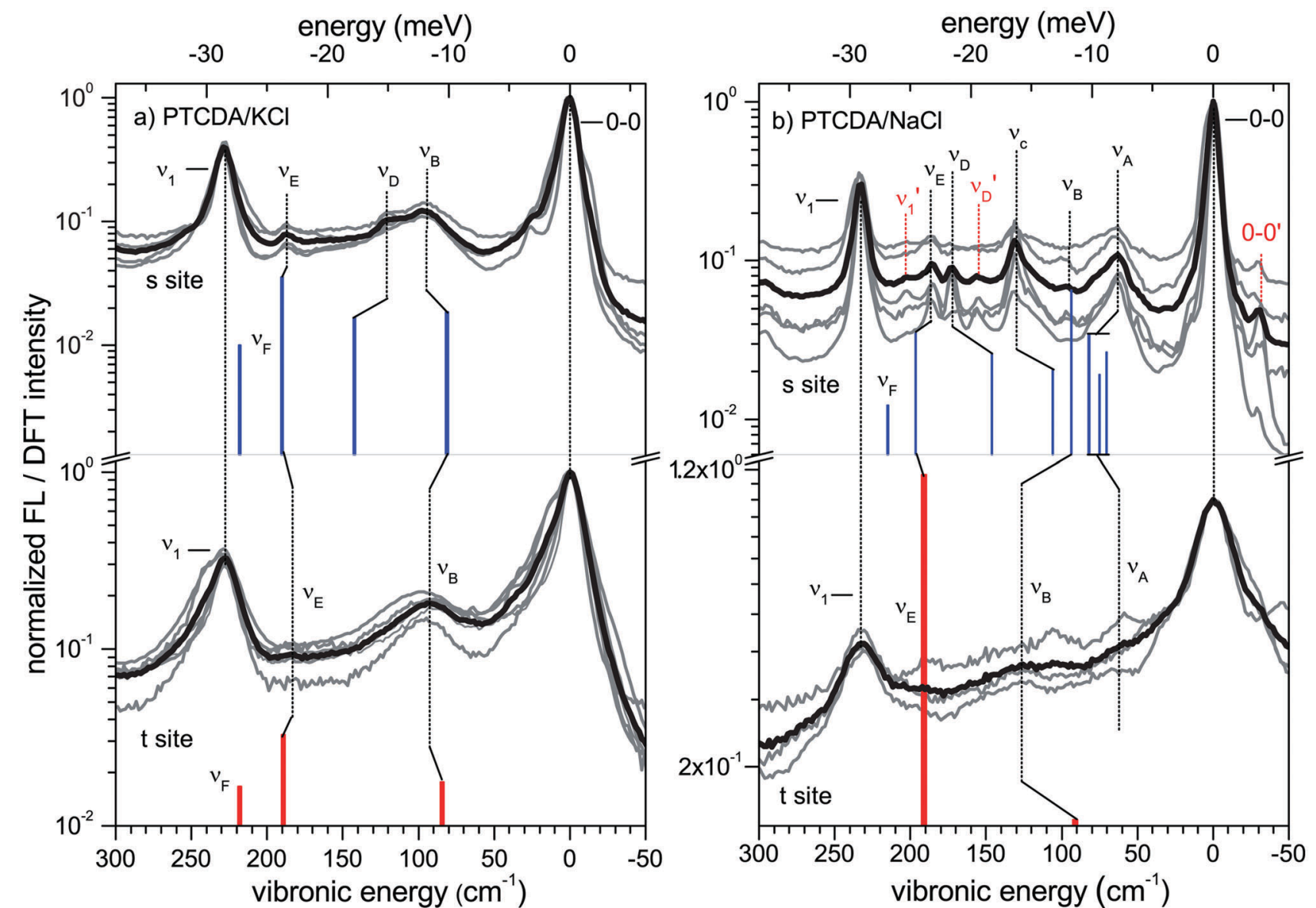

Fig. 2 Comparison of the low vibrational energy range of the fluorescence (FL) spectra of PTCDA molecules on $t$ sites and $s$ sites (shifted vertically for clarity) on $\mathrm{KCl}$ (a) and $\mathrm{NaCl}$ (b). All spectra were aligned and normalized at the positions of the $0-0$ lines. These are at $20011 \pm 20 \mathrm{~cm}^{-1}$ and $20146 \pm 20 \mathrm{~cm}^{-1}$ for the $t$ and s site spectra on $\mathrm{KCl}$, and at $19755 \pm 20 \mathrm{~cm}^{-1}$ and $19906 \pm 20 \mathrm{~cm}^{-1}$ on NaCl. In order to emphasize small features in the spectra logarithmic scales were used. The heavy black line is the average of several spectra measured for different independent preparations; these are shown in addition as thin gray lines in order to illustrate the overall variations of the spectra. In addition to the experimental data, the energies of vibrational modes calculated by using DFT are indicated by stick spectra. The lengths of the sticks represent the size of the calculated respective Franck-Condon factors which were adjusted to the experimental spectra such that the intensity of the mode $\nu_{1}$ is correctly fitted. They are plotted on the same logarithmic scales as the fluorescence intensities, except for the $t$ site on $\mathrm{NaCl}$, where they have been multiplied by a factor of 10 . Red sticks represent vibrational modes belonging to the totally symmetric representation $A_{1}$, blue sticks represent modes of the $A$. The small peak at $25 \mathrm{~cm}^{-1}$ in the $\mathrm{KCl}$ s site spectra is not a vibrational peak, but most likely due the $0-0$ line of a minority of molecules adsorbed on a metastable (X10), as we described in ref. 45 .

$\left(\leq 19 \mathrm{~cm}^{-1}\right)$ from those that were reported earlier in ref. 19. The vibrational energies of the $\mathrm{NaCl} t \mathrm{t}$ sites are tabulated here for the first time on the basis of recent spectra. (An earlier spectrum can be found in ref. 29.) The energies of all these

\$ We note that in ref. 29 the position the $0-0$ transitions for PTCDA on $\mathrm{NaCl} t$ site was reported at a slightly different energy of $19680 \mathrm{~cm}^{-1}$. We attribute this to a smaller experimental resolution (grating of 300 grooves per $\mathrm{mm}$ ) and a different excitation wavelength of $476.5 \mathrm{~nm}$ used in the earlier experiment. vibrational modes are very similar to those measured for PTCDA by Stienkemeier and coworkers, ${ }^{14,16}$ using the helium nanodroplet isolation technique. ${ }^{46}$ Taken all together, this demonstrates that the modes above $230 \mathrm{~cm}^{-1}$ do not couple to substrate phonons and are thus not modified by the substrate bonding. The former aspect is understandable because the phonon modes of $\mathrm{KCl}$ and $\mathrm{NaCl}$ have energies below $200 \mathrm{~cm}^{-1}$ (ref. 47 and 48 ) and do thus not mix with the vibrational modes above $230 \mathrm{~cm}^{-1}$.

Table 1 (shown on the next page) Energies of the vibrational peaks in the FL spectra of PTCDA at terrace (t) and step (s) sites on (100) oriented NaCl and $\mathrm{KCl}$ films. These energies are averaged values obtained from sets of spectra measured for different sample preparations. These are shown in Fig. 2 for the low energy range. Exemplary spectra of the s sites for the full energy range are given in Fig. 3. Energies calculated using DFT are also listed. An assignment of all experimentally observed vibrational energies to the contributing fundamental modes is given in the first column. The assignment in brackets refers to that used in ref. 36. For experimental modes which result from combinations of modes the energies of the contributing fundamental modes and the respective sums are given in brackets in the respective columns. For some peaks, the assignments are not unique and two alternatives are given. All values are given in $\mathrm{cm}^{-1}$. The "-" denotes that the modes have experimentally not been detected or possess intensities below 0.001 . An exception is the mode calculated using DFT at $56 \mathrm{~cm}^{-1}$ discussed in the text 


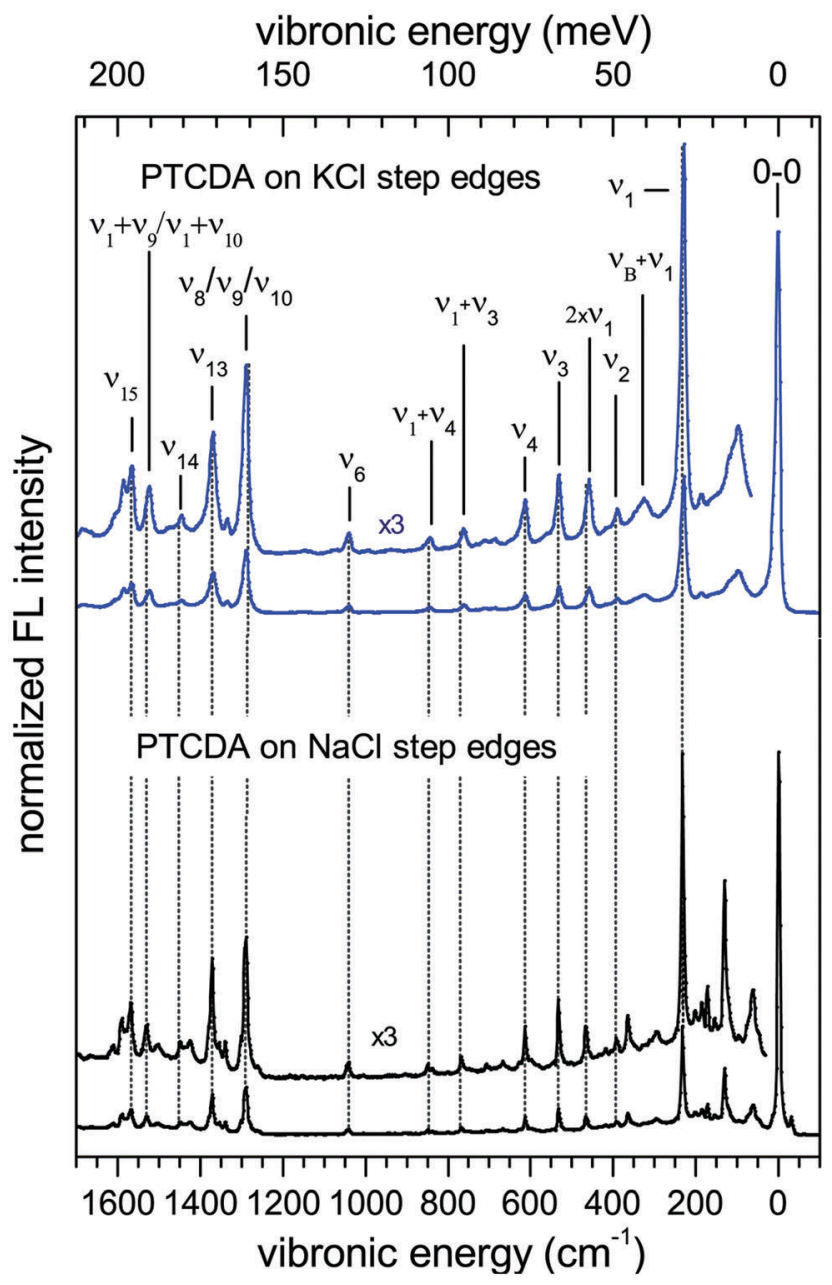

Fig. 3 Comparison of overview fluorescence (FL) spectra of PTCDA molecules at step edge sites on $\mathrm{KCl}$ (top) and $\mathrm{NaCl}$ (bottom). The spectra were aligned at their $0-0$ transitions (20162 $\pm 20 \mathrm{~cm}^{-1}$ and $19906 \pm 20 \mathrm{~cm}^{-1}$ ). The positions of the vibrational modes are also summarized in Table 1.

\subsection{Modifications of the surface induced modes due to the} site transition

Coming back to Fig. 2, we make the following observations. Upon the migration of the molecules from the $t$ sites to s sites, the spectra change in the range of the SIM, both for the $\mathrm{KCl}$ and the $\mathrm{NaCl}$ surface. In the spectra of the $t$ sites we see the following peaks: on $\mathrm{KCl}$, there is one clear broad peak at $94 \mathrm{~cm}^{-1}$ and a small peak at $185 \mathrm{~cm}^{-1}$; on $\mathrm{NaCl}$, we can identify three broad peaks at $57 \mathrm{~cm}^{-1}, 127 \mathrm{~cm}^{-1}$, and $186 \mathrm{~cm}^{-1}$. Quite differently, the spectra of the s sites show more and sharper peaks. On $\mathrm{KCl}$, we find three peaks; on $\mathrm{NaCl}$ we find seven peaks. For convenience we label these experimentally determined peaks by $\nu_{\mathrm{A}}$ to $\nu_{\mathrm{E}}$ and by $\nu_{1}^{\prime}$ and $\nu_{\mathrm{D}}{ }^{\prime}$ (see Fig. 2). Hereby the two small peaks $\nu_{1}{ }^{\prime}$ and $\nu_{\mathrm{D}}{ }^{\prime}$ (only seen on $\mathrm{NaCl}$ ) belong to a second minority species of PTCDA, evidenced by a second small blue shifted $0-0$ transition, $30 \mathrm{~cm}^{-1}$ above the $0-0$ transition of the majority species. The origin of this second species is not clear yet and we will thus not consider the related peaks any further. Hence, we have to compare three SIM on $\mathrm{NaCl}$ to two
SIM on $\mathrm{KCl}$ for the t site spectra, and five SIM on $\mathrm{NaCl}$ to three $\mathrm{SIM}$ on $\mathrm{KCl}$ for the $\mathrm{s}$ site spectra. In summary we find differences between the $t$ and s site spectra on both surfaces, and differences in the respective site spectra for $\mathrm{KCl}$ and $\mathrm{NaCl}$. Thus two questions arise at this point. First, why do we find different SIM for the $\mathrm{t}$ and s sites, and, secondly, how can we explain the differences in the respective spectra on $\mathrm{KCl}$ and $\mathrm{NaCl}$ ?

We start with the discussion of the first question: the difference between $t$ and s site spectra. Evidently the symmetry of the adsorption site of the molecules on the terrace is $C_{2 \mathrm{v}}$. This can be seen from the hard sphere models in Fig. 1. As a consequence the symmetry of the molecular geometry is also $C_{2 \mathrm{v}}$. When the molecules are adsorbed at the step edges, the symmetry of the adsorption site is reduced to $C_{1}$ due to the asymmetric embedding of the molecules at the step edges. This can also be derived from the hardsphere model of the $t$ sites in Fig. 1. As a consequence of the reduced symmetry of the adsorption site we can expect and confirm by the DFT calculations a reduced symmetry of the molecular geometry, too. This has an implication on the vibrational modes that can couple to the optical transition. For the s site with reduced symmetry we can expect that modes which formerly did not couple to the optical transition in the $C_{2 \mathrm{v}}$ symmetry. Namely, these are all modes not belonging to the $\mathrm{A}_{1}$ representation of $C_{2 \mathrm{v}}$, but belonging to the $A_{2}, B_{1}$, and $B_{2}$ representations. They can now couple to the optical transition, because, for the $C_{1}$ symmetry, there exists only the fully symmetric representation A. The reduction of the symmetry is thus the most important mechanism for the changes in the spectra by the appearance of additional modes.

In addition, differential shifts between the energies of the modes that couple to the transition for both the $t$ and $s$ sites can be expected because of the different interactions of the molecule with surface atoms on the $t$ and s sites. Furthermore, the intensities of these modes may vary for the $t$ and $s$ sites, because the respective FC factors can change due to different structures of the ground and excited states on the two sites. The above described effects can also explain the variation between the spectra on $\mathrm{KCl}$ and $\mathrm{NaCl}$. Due to the different molecule surface interactions different mode energies can be expected. This behavior is in contrast to that of the high energy vibrational modes which are nearly not effected by the bonding of the molecule to the surface, as we reported in ref. 19 and 29.

Moreover, different distortion patterns will be reflected in different FC factors and respective mode intensities. The observation of a larger number of modes on $\mathrm{NaCl}$ compared to $\mathrm{KCl}$ can be explained by the fact that, on $\mathrm{KCl}$, some modes couple only very weakly to the transition and are thus not observed, because due to smaller molecular distortions the respective FC factors are too small to cause peaks of detectable intensities.

\subsection{Interpretation of the SIM on the basis of DFT calculations}

The vibrational modes of the free and non-distorted PTCDA molecule with $D_{2 \mathrm{~h}}$ symmetry were calculated by means of DFT 
by Tautz et $a .^{1}$ The five lowest modes, in the energy range below $200 \mathrm{~cm}^{-1}$, were found at $36 \mathrm{~cm}^{-1}\left(\mathrm{~B}_{3 \mathrm{u}}\right), 111 \mathrm{~cm}^{-1}\left(\mathrm{~A}_{\mathrm{u}}\right)$, $136 \mathrm{~cm}^{-1}\left(\mathrm{~B}_{2 \mathrm{u}}\right), 174 \mathrm{~cm}^{-1}\left(\mathrm{~B}_{3 \mathrm{u}}\right)$, and $195 \mathrm{~cm}^{-1}\left(\mathrm{~B}_{3 \mathrm{u}}\right)$ (see Table 1 in ref. 1). The $36 \mathrm{~cm}^{-1}$ and the $111 \mathrm{~cm}^{-1} / 174 \mathrm{~cm}^{-1}$ modes are short and long axis folding modes, respectively. The $36 \mathrm{~cm}^{-1}$ mode corresponds to an arch-like distortion of the molecule with a vertical displacement of the anhydride groups with respect to the molecular plane. ${ }^{49}$ Evidently, the energies of these modes which are calculated for the free molecule will be modified by the bonding to the $\mathrm{NaCl}$ and $\mathrm{KCl}$ substrates. Such an energy shift due to the surface bonding was observed experimentally for PTCDA on the Ag(110) surface in HREELS spectra: the mode at $213 \mathrm{~cm}^{-1}$ of the multilayer shifts to $204 \mathrm{~cm}^{-1}$ in the submonolayer due to the surface bonding. This mode was assigned to the mode calculated using DFT at $195 \mathrm{~cm}^{-1}$ for the free molecule. From these DFT calculations we can conclude that vibrational peaks of PTCDA have to be expected in the considered energy range up to $200 \mathrm{~cm}^{-1}$. However, the so computed energies do of course not explain the here observed vibrational energies. In addition, because they refer to the free molecule, they do not yield an interpretation of the differences between the energies on the $\mathrm{KCl}$ and $\mathrm{NaCl}$ surfaces. For this reason we have computed the vibrational modes of PTCDA adsorbed on the $\mathrm{KCl}$ and $\mathrm{NaCl}(100)$ surfaces by means of DFT on our own. In these calculations, the PTCDA molecule at a terrace site ( $\mathrm{t}$ site) had the corresponding $C_{2 \mathrm{v}}$ symmetry. As described in Section 2.2 the PTCDA molecule at the $\mathrm{s}$ site was modeled by an adsorbate/cluster model with a deliberately reduced symmetry, namely $C_{1}$, as it is expected at the s sites. Even though we used this strongly simplified model for the s site and only allowed for molecular vibrations located on the molecule, these calculations showed how the reduced symmetry of the adsorption site causes the appearance of additional vibronic modes in the spectra, in agreement with what is observed experimentally: namely, all totally symmetric $\mathrm{A}_{1}$ modes of the $C_{2 \mathrm{v}}$ symmetry can contribute to the t site spectra (red sticks in Fig. 2), and all modes of the $C_{1}$ symmetry, which are all totally symmetric and belong to the A representation, (blue sticks in Fig. 2) can contribute to the s site spectra.

Our calculations took full account of the interactions of the molecule with the substrate and of the induced distortions (out-of-plane bending) of the molecule in the ground state $\left(\mathrm{S}_{0}\right)$. Both aspects are expected to have impact on the energies of the vibrational modes. In a second step, in order to evaluate which of the vibrational modes couple effectively to the optical transition, the FC factors of the respective vibrational modes were calculated. This involved the additional computation of the geometry of the molecule in the excited $S_{1}$ state on the surfaces. This part of the calculation and the derived geometries were described earlier in ref. 23. As noted in Section 2.2, the vibrations of the surface ions were neglected, which is an approximation of the real situation. However, this procedure safeguards that only those modes which are mainly located on the molecule, and which are of interest here, were calculated. In Fig. 2, the positions of all calculated modes, which exhibit intensities above a certain cut-off of 0.001 , are depicted as stick spectra with red and blue lines. The lengths of the sticks indicate the respective FC factors. The corresponding distortion patterns, are illustrated in Fig. 4 for the relevant modes. The energies of these DFT calculated modes are included in Table 1 for comparison.

We start with the inspection of the spectra on KCl (Fig. 2(a)). There we find a rather good one-to-one agreement in the energies of the calculated and experimentally measured modes with deviations of $24 \mathrm{~cm}^{-1}$ at the most. The calculated modes and the assignment to the experimental modes $\left(\nu_{\mathrm{B}}, \nu_{\mathrm{D}}\right.$, and $\left.\nu_{\mathrm{E}}\right)$ are illustrated in Fig. 4. In the s site spectrum, one additional mode $\left(\nu_{\mathrm{D}}\right)$ is observed in comparison with the t site spectrum in the experimental and DFT data ( $c f$. Fig. $4,142 \mathrm{~cm}^{-1}$ ). This mode stems from a mode of the $\mathrm{B}_{2}$ representations of the $C_{2 \mathrm{v}}$ symmetry which now, for the $C_{1}$ symmetry, projects onto the A representation and is thus observed for the transition in the reduced symmetry of the $s$ site. In addition, from the DFT calculations we found a mode (" $\nu_{\mathrm{F}}$ ") (for both the $C_{2 \mathrm{v}}$ and $C_{1}$ symmetry), which is not observed in the experimental spectra. The calculated intensities of this mode are very small $(0.016$ (0.100) for $\left.C_{2 \mathrm{v}}\left(C_{1}\right)\right)$. Therefore this mode is not visible in the experimental spectra, or it is hidden under the strong $\nu_{1}$ mode.

For $\mathrm{NaCl}$ we start with the discussion of the $\mathrm{t}$ site spectrum (Fig. 2(b), bottom spectrum). Here we find three DFT calculated modes belonging to the $\mathrm{A}_{1}$ representation of the $C_{2 \mathrm{v}}$ symmetry (see Fig. 4). The calculated energies of the modes at $91 \mathrm{~cm}^{-1}$ and $191 \mathrm{~cm}^{-1}$ fit roughly those of the two experimental modes $\nu_{\mathrm{B}}\left(127 \mathrm{~cm}^{-1}\right)$, and $\nu_{\mathrm{E}}\left(186 \mathrm{~cm}^{-1}\right)$ observed in the experiment. However, the intensities of the calculated modes $\nu_{\mathrm{B}}$ and $\nu_{\mathrm{E}}$ come out too small by factors of about 25 and 3, respectively. The same is true for the mode $\nu_{\mathrm{A}}\left(57 \mathrm{~cm}^{-1}\right)$; the corresponding mode calculated by DFT is in good agreement at $57 \mathrm{~cm}^{-1}$, but exhibits a much too small intensity (and was thus not included in Fig. 2(b)). We suppose that the reason for these discrepancies in the intensities is that we neglect the vibrational motions of the substrate atoms in our model calculations. The amplitudes of these increase in particular at low energies.

The s site spectrum shows five peaks (Fig. 2(b), upper spectrum). The peak at the lowest energy $\left(\nu_{\mathrm{A}}\right)$ is strongly enhanced with respect to the $\mathrm{t}$ site spectrum. This is explained by the contributions of three unresolved additional modes which couple only in the $C_{1}$ symmetry to the $\nu_{\mathrm{A}}$ peak. These modes stem from modes belonging to the $\mathrm{A}_{1}, \mathrm{~B}_{1}$, and $\mathrm{A}_{2}$ representations of the $C_{2 \mathrm{v}}$ symmetry, projecting into the A representation of the $C_{1}$ symmetry. The peak $\nu_{\mathrm{B}}$ is observed in both the s site spectrum and in the t spectrum and is shifted by $31 \mathrm{~cm}^{-1}$ to smaller vibrational energies for the latter. The peak is well fitted by the theory. The peak $\nu_{\mathrm{E}}$ is contained in the $\mathrm{s}$ site spectrum as in the $\mathrm{t}$ site spectrum at similar positions (within $1 \mathrm{~cm}^{-1}$ ) and is also fitted by theory. The peaks $\nu_{\mathrm{C}}$ and $\nu_{\mathrm{D}}$ are only visible in the s site spectrum (not in the t site spectrum), because these modes belong to the $\mathrm{A}_{2}\left(\nu_{\mathrm{C}}\right)$ and $\mathrm{B}_{1}\left(\nu_{\mathrm{D}}\right)$ representations of the $C_{2 \mathrm{v}}$ symmetry and thus do not couple. The corresponding calculated peaks come out at too small energies (about $25 / 27 \mathrm{~cm}^{-1}$ ). The DFT calculated mode at $215 \mathrm{~cm}^{-1}\left(\nu_{\mathrm{F}}\right)$ cannot be observed in the FL spectra, as found for PTCDA on $\mathrm{KCl}$.

One important conclusion is that in the $\mathrm{NaCl}$ s site spectrum two additional modes $\left(\nu_{\mathrm{C}}\right.$ and $\left.\nu_{\mathrm{D}}\right)$ couple to the transition with respect to the $\mathrm{t}$ site spectrum instead of only one additional 
$\nu_{\mathrm{A}}$

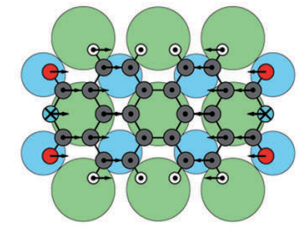

$\mathrm{NaCl} / \mathrm{C}_{2 \mathrm{v}}$ $56 \mathrm{~cm}^{-1} /<0.001$

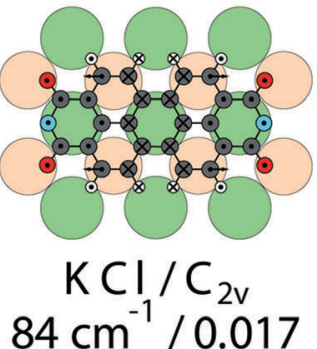

$\nu_{\mathrm{B}}$

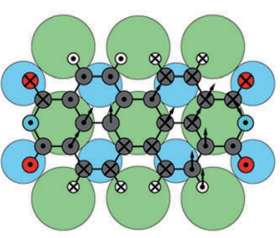

$\mathrm{NaCl} / \mathrm{C}_{1}$
$106 \mathrm{~cm}^{-1} / 0.020$

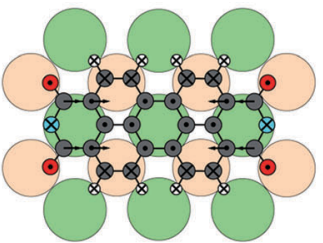

$\mathrm{KCl} / \mathrm{C}_{2 \mathrm{v}}$ $189 \mathrm{~cm}^{-1} / 0.033$

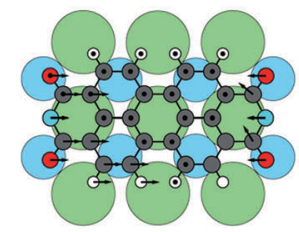

$\mathrm{NaCl} / \mathrm{C}_{1}$ $70 \mathrm{~cm}^{-1} / 0.026$

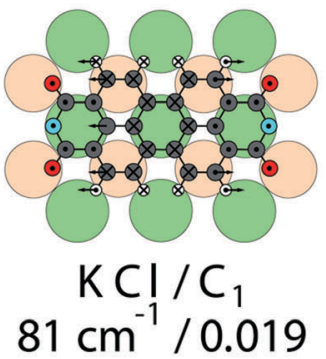

$\nu_{\mathrm{D}}$

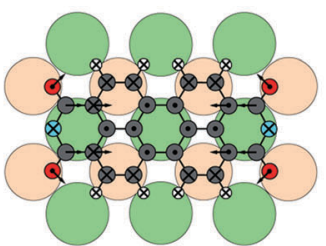

$\mathrm{KCl} / \mathrm{C}_{1}$ $190 \mathrm{~cm}^{-1} / 0.035$

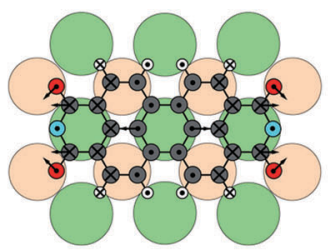

$\mathrm{KCl} / \mathrm{C}_{1}$

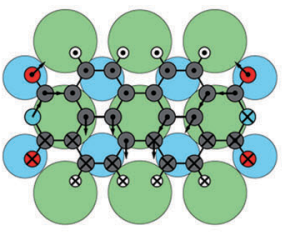

$\mathrm{NaCl} / \mathrm{C}_{1}$
$75 \mathrm{~cm}^{-1} / 0.019$

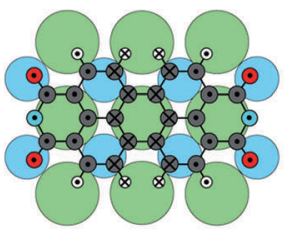

$\mathrm{NaCl} / \mathrm{C}_{2 \mathrm{v}}$ $91 \mathrm{~cm}^{-1} / 0.012$
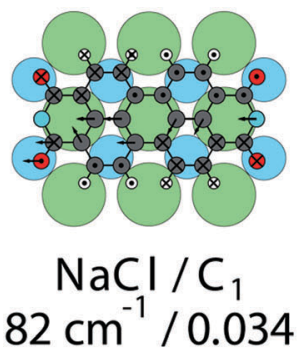

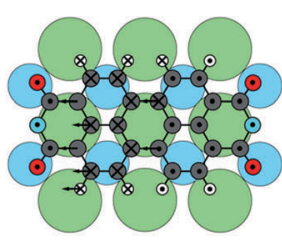

$\mathrm{NaCl}^{-1} \mathrm{C}_{1}$
$94 \mathrm{~cm}^{-1} / 0.065$
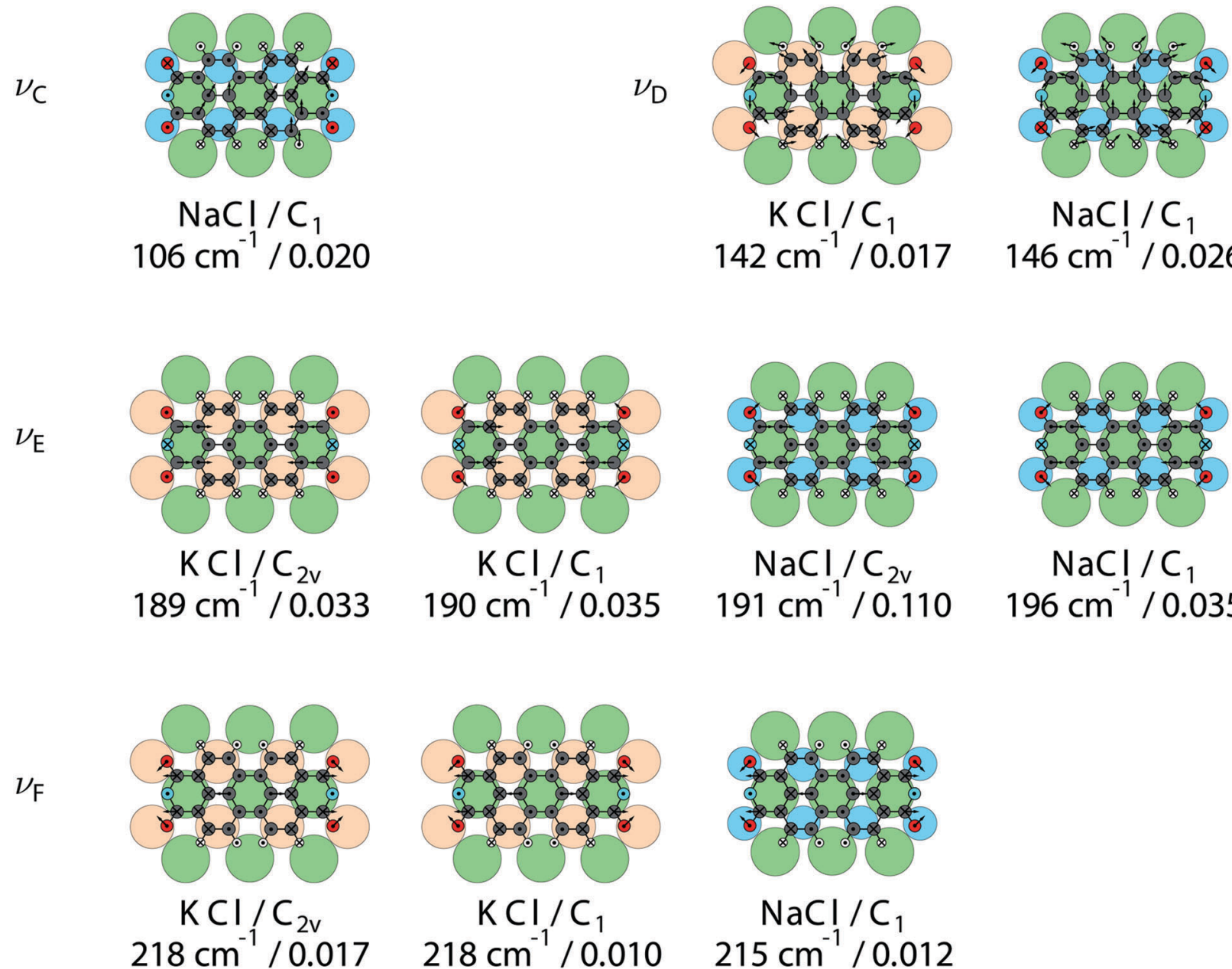

$142 \mathrm{~cm}^{-1} / 0.017$
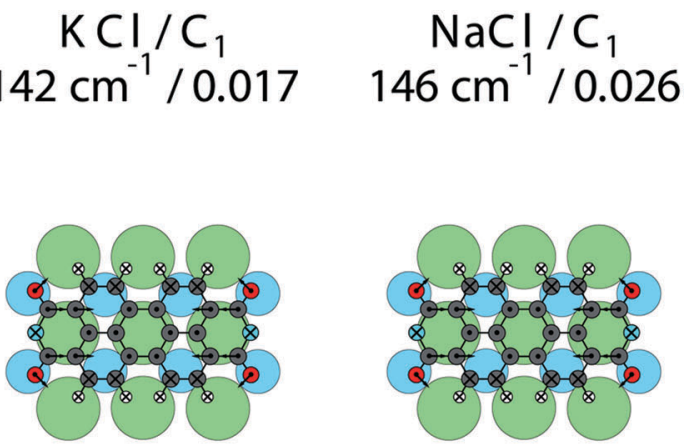

$\mathrm{NaCl} / \mathrm{C}_{2 \mathrm{v}}$ $191 \mathrm{~cm}^{-1} / 0.110$

$\mathrm{NaCl} / \mathrm{C}_{1}$ $196 \mathrm{~cm}^{-1} / 0.035$

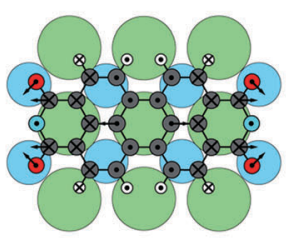

$\mathrm{NaCl} / \mathrm{C}_{1}$ $215 \mathrm{~cm}^{-1} / 0.012$

Fig. 4 Illustrations of the respective low energy vibrational modes as calculated using DFT for the tow different symmetries $\left(C_{2 v} / C_{1}\right)$ of the PTCDA on $\mathrm{KCl}(100)$ and $\mathrm{NaCl}(100)$. The arrows, crosses and bold points represent the movement of the atoms during the vibrations. The bold points and crosses indicate upwards respectively downwards out-of-plane movements. In addition the calculated energies and the respective FC factors calculated by theory are given. Note that step edge atoms have been omitted for $C_{1}$ symmetry for clarity. 
mode as it is observed in the KCl s site spectrum $\left(\nu_{\mathrm{D}}\right)$. This can be understood as an effect from a stronger deviation of the molecular symmetry at the steps from the $C_{2 \mathrm{v}}$ symmetry on $\mathrm{NaCl}$ compared to the situation on $\mathrm{KCl}$. This is plausible because the vacancies formed by the substrate ions around one end of the molecule (see Fig. 1) have a smaller size on $\mathrm{NaCl}$ (due to the smaller lattice constant) and thus lead to stronger lateral interactions between the ions of the step and the "embedded" anhydride group of the PTCDA. These interactions obviously reduce the molecular $C_{2 \mathrm{v}}$ symmetry more strongly causing a stronger coupling of the above modes to the optical transition. We will look at this aspect again, when we discuss the mode patterns in the next section. This effect of lateral interactions with the ions of the step on the molecular symmetry was also discussed for the shift in the energy of the electronic transition from the $t$ to the sites. ${ }^{28,45}$

\subsection{Interpretation with aid of the distortion patterns}

Schematic diagrams of the relevant modes are illustrated in Fig. 4. The totally symmetric modes of the $C_{2 \mathrm{v}}$ symmetry $\left(\mathrm{A}_{1}\right)$ correspond to short axis $\left(\nu_{\mathrm{A}}, \nu_{\mathrm{B}}\right)$ and long axis $\left(\nu_{\mathrm{E}}\right)$ folding modes. The modes, which appear additionally for the s sites, contain elements of rotations that can be understood as frustrated rotations of the free molecule. These modes couple to the spectra only, when the symmetry is reduced due to the presence of the step edge, as illustrated in Fig. 1 . The mode $\nu_{\mathrm{D}}$ seen on both surfaces exhibits a significantly smaller vibrational energy on $\mathrm{KCl}$ compared to $\mathrm{NaCl}$ (see Fig. 2). We explain this by the stronger interaction of the PTCDA with the surface ions embedding one part of the molecule on $\mathrm{NaCl}$ compared to $\mathrm{KCl}$. This effect is in particular seen from the stronger out-of-plane distortion of the molecule visible in the side views in Fig. 1.

For $\mathrm{NaCl}$ we find a larger number of the non-symmetric modes to couple to the transition for the s site. As noted above, we explain this by larger FC factors of these modes on $\mathrm{NaCl}$ compared to $\mathrm{KCl}$ as it is suggested by the DFT calculation. Since the FC factors reflect the displacements of the molecular coordinates under the optical excitation, we can conclude from the stronger coupling of these modes that the respective displacements on $\mathrm{NaCl}$ are significantly larger than on KCl. We ascribe this to the smaller geometric size of the vacancy at the step and the thus stronger electrostatic lateral interactions of the charged anhydride groups with the surrounding surface ions. (We note that this is not modelled by the DFT calculations so far, because these do not describe the step sites, yet.) Under the optical transition the local charge distribution on the PTCDA is altered and thus the electrostatic interactions between partially charged molecular groups, e.g. the carboxylic $\mathrm{O}$ atoms, and step edge ions, e.g., the $\mathrm{K}^{+}$cations, are altered. Stronger interactions thus will cause stronger displacements $\Delta q_{i}$ on $\mathrm{NaCl}$ compared to $\mathrm{KCl}$, although the changes in the charge distributions may be very similar.

Finally we have to explain the difference in the differential shifts from the terrace to the step for $\mathrm{KCl}$ and $\mathrm{NaCl}$. In general the shifts are only small. However, for the mode $\nu_{\mathrm{B}}$ we see a large difference, namely a shift of $-31 \mathrm{~cm}^{-1}$ on $\mathrm{NaCl}$, while on
$\mathrm{KCl}$, the shift is only $+3 \mathrm{~cm}^{-1}$. This mode is attributed to a short axis folding mode and illustrated in Fig. 4 . It appears to be very sensitive to the presence of the steps and is thus shifted much stronger on $\mathrm{NaCl}$ than on $\mathrm{KCl}$.

\section{Final discussion}

Our results demonstrate how fluorescence spectroscopy can be used to identify structural differences in the adsorption sites of a molecule. This information is most helpful for further interpreting different adsorption sites which have been previously identified from their optical transitions at different energies. The intensities of the considered low energy vibrational modes scale with the change in the structure of the molecule upon the optical excitation via the Franck-Condon factors. In the present case, the $S_{0} / S_{1}$ transition corresponds to an excitation of an electron from the HOMO to LUMO and induces a rearrangement of the charges on the PTCDA molecule. The change in the charge density of the free molecule has been reported for instance by Scholz et al. ${ }^{50}$ The charge redistribution causes a modification of the electrostatic interactions of the molecule with the surface ions and a concomitant change of the molecular geometry upon the optical excitation. In principle the calculation of the FC factors of the vibrational modes by means of DFT for an anticipated adsorption site in combination with comparison with the experimentally observed intensities, which are proportional to the respective FC factors, can yield detailed structural information about adsorption sites. For the present example, DFT calculations have been only available for the terrace sites, yet. Nevertheless there is no principal restriction for calculations of the vibrational modes and the respective FC factors for molecules at the step sites.

The energetic resolution of the vibrational modes, i.e. their line shape, is given by the inhomogeneous line broadening of the electronic transition ${ }^{45}$ due to the small variations in the details of the adsorption sites, e.g., the arrangement of additional ion pairs of $\mathrm{Na}^{+} \mathrm{Cl}^{-}$and $\mathrm{K}^{+} \mathrm{Cl}^{-}$on the two sides of the molecule. The full width at half maximum of the vibrational peaks of the s site spectra $\left(\sim 10 \mathrm{~cm}^{-1}\right)$ is comparable with that obtained in good HREELS spectra. ${ }^{1,51}$ However, fundamentally different from HREELS the resolution of fluorescence (FL) spectra is not limited by the instrumental setup, but by the inhomogeneous line broadening related to the sample. In principle it would be possible to deconvolute the line shape of the pure electronic transition out of the vibronic peaks. When comparing HREELS and FL spectroscopy one should also bear in mind that, in general, both methods are sensitive to vibrational modes of different symmetry, which in our case agree due to the low symmetry of the adsorption complex. The sensitivity of FL spectroscopy is at least equally high as that of HREELS and allows one to investigate small coverages below few percent of a monolayer. Complementary to HREELS, FL spectroscopy can be performed on insulating wide-band gap substrates, but not on metallic surfaces. 


\section{Summary}

We investigated the low energy vibrational modes of isolated PTCDA molecules, adsorbed on the $\mathrm{KCl}$ and $\mathrm{NaCl}(100)$ surfaces, which couple to the emission in fluorescence spectra. The coupling of these modes can only be explained by a significant distortion of the PTCDA in the ground $\left(\mathrm{S}_{0}\right)$ or excited state $\left(\mathrm{S}_{1}\right)$ from its planar gas phase geometry induced by the adsorption on the surfaces. Remarkably we found the appearance of new vibrational modes in the spectra recorded for PTCDA at step edge sites when comparing these to spectra of PTCDA adsorbed on the terrace sites. This can be understood by a further reduction of the symmetry of the molecule at the step edge sites with respect to the terrace sites by the interaction with the step edge ions. The effect is less pronounced on $\mathrm{KCl}$ compared to $\mathrm{NaCl}$ and can be explained by the difference between the lattice constants and the resulting stronger bonding and distortion of the molecule on $\mathrm{NaCl}$ compared to $\mathrm{KCl}$.

\section{Acknowledgements}

This work was supported by the DFG under the projects SO407/8-1, SCHM1361/21, and DACH 1958. MH acknowledges a scholarship by the Studienstiftung des deutschen Volkes. We further thank the Paderborn Center for Parallel Computing, $\mathbf{P C}^{2}$, and the Leibniz University IT Services (former Regionales Rechenzentrum für Niedersachsen [RRZN]) for providing computational resources.

\section{References}

1 F. S. Tautz, S. Sloboshanin, J. Schaefer, R. Scholz, V. Shklover, M. Sokolowski and E. Umbach, Phys. Rev. B: Condens. Matter Mater. Phys., 2000, 61, 16933-16947.

2 M. Müller, A. Langner, O. Krylova, E. Le Moal and M. Sokolowski, Appl. Phys. B: Lasers Opt., 2011, 105, 67-79.

3 L. Nony, F. Bocquet, F. Para, F. Cherioux, E. Duverger, F. Palmino, V. Luzet and C. Loppacher, Beilstein J. Nanotechnol., 2012, 3, 285-293.

4 Q. Jia, W. Ji, S. A. Burke, H.-J. Gao, P. Grütter and H. Guo, Phys. Chem. Chem. Phys., 2016, 18, 11008-11016.

5 O. Pakarinen, J. Mativetsky, A. Gulans, M. Puska, A. Foster and P. Grütter, Phys. Rev. B: Condens. Matter Mater. Phys., 2009, 80, 085401-085405.

6 B. Hoff, M. Gingras, R. Peresutti, C. R. Henry, A. S. Foster and C. Barth, J. Phys. Chem. C, 2014, 118, 14569-14578.

7 O. Bauer, G. Mercurio, M. Willenbockel, W. Reckien, C. Schmitz, B. Fiedler, S. Soubatch, T. Bredow, F. S. Tautz and M. Sokolowski, Phys. Rev. B: Condens. Matter Mater. Phys., 2012, 86, 235431.

8 M. Müller, A. Paulheim, A. Eisfeld and M. Sokolowski, J. Chem. Phys., 2013, 139, 044302.

9 M. Müller, J. Ikonomov and M. Sokolowski, Surf. Sci., 2011, 605, 1090-1094.

10 T. Dienel, C. Loppacher, S. C. B. Mannsfeld, R. Forker and T. Fritz, Adv. Mater., 2008, 20, 959.
11 D. R. Zahn, G. N. Gavrila and G. Salvan, Chem. Rev., 2007, 107, 1161-1232.

12 K. Akers, R. Aroca, A. M. Hor and R. O. Loutfy, J. Chem. Phys., 1987, 91, 2954-2959.

13 R. Scholz, A. Y. Kobitski, T. U. Kampen, M. Schreiber, D. R. T. Zahn, G. Jungnickel, M. Elstner, M. Sternberg and T. Frauenheim, Phys. Rev. B: Condens. Matter Mater. Phys., 2000, 61, 13659-13669.

14 M. Dvorak, M. Müller, T. Knoblauch, O. Bünermann, A. Rydlo, S. Minniberger, W. Harbich and F. Stienkemeier, J. Chem. Phys., 2012, 137, 164302.

15 M. Wewer and F. Stienkemeier, Phys. Chem. Chem. Phys., 2005, 7, 1171-1175.

16 M. Wewer and F. Stienkemeier, J. Chem. Phys., 2004, 120, 1239-1244.

17 E. Condon, Phys. Rev., 1926, 28, 1182-1201.

18 H. Aldahhak, W. G. Schmidt and E. Rauls, Surf. Sci., 2015, 641, 278-281.

19 M. Müller, A. Paulheim, C. Marquardt and M. Sokolowski, J. Chem. Phys., 2013, 138, 064703.

20 Q. Guo, A. Paulheim, M. Sokolowski, H. Aldahhak, E. Rauls and W. G. Schmidt, J. Phys. Chem. C, 2014, 118, 29911-29918.

21 H. Karacuban, S. Koch, M. Fendrich, T. Wagner and R. Möller, ACS Nano, 2011, 22, 295305.

22 H. Aldahhak, W. G. Schmidt and E. Rauls, Surf. Sci., 2013, 617, 242-248.

23 M. Hochheim and T. Bredow, J. Comput. Chem., 2015, 36, 1805-1811.

24 F. Mohn, J. Repp, L. Gross, G. Meyer, M. S. Dyer and M. Persson, Phys. Rev. Lett., 2010, 105, 266102.

25 J. D. H. Donnay, W. P. Mason and E. A. Wood, American Institute of Physics Handbook, McGraw-Hill Book Company, New York, 3rd edn, 1972.

26 D. Schlettwein, A. Back, B. Schilling, T. Fritz and N. R. Armstrong, Chem. Mater., 1998, 10, 601-612.

27 M. Möbus, N. Karl and T. Kobayashi, J. Cryst. Growth, 1992, 116, 495-504.

28 A. Paulheim, M. Müller, C. Marquardt and M. Sokolowski, Phys. Chem. Chem. Phys., 2013, 15, 4906-4913.

29 M. Müller, E. Le Moal, R. Scholz and M. Sokolowski, Phys. Rev. B: Condens. Matter Mater. Phys., 2011, 83, 241203.

30 E. Le Moal, M. Müller, O. Bauer and M. Sokolowski, Surf. Sci., 2009, 603, 2434-2444.

31 J. P. Perdew, K. Burke and M. Ernzerhof, Phys. Rev. Lett., 1996, 77, 3865-3868.

32 S. Grimme, J. Antony, S. Ehrlich and H. Krieg, J. Chem. Phys., 2010, 132, 154104-154119.

33 S. Grimme, S. Ehrlich and L. Goerigk, J. Comput. Chem., 2011, 32, 1456-1465.

34 J. P. Perdew, K. Burke and M. Ernzerhof, Phys. Rev. Lett., 1997, 78, 1396.

35 R. Dovesi, V. Saunders, C. Roetti, R. Orlando, C. M. ZicovichWilson, F. Pascale, B. Civalleri, K. Doll, N. Harrison, I. Bush, P. D'Arco, M. Llunell, M. Causà and Y. Noël, CRYSTAL14 user's manual, University of Torino, Torino, 2014. 
36 M. Hochheim, A. Paulheim, M. Sokolowski and T. Bredow, J. Phys. Chem. C, 2016, 120, 24240-24249.

37 R. Dovesi, C. Roetti, C. Freyria-Fava, M. Prencipe and V. R. Saunders, Chem. Phys., 1991, 156, 11-19.

38 B. Civalleri, A. M. Ferrari, M. Llunell, R. Orlando, M. Mérawa and P. Ugliengo, Chem. Mater., 2003, 15, 3996-4004.

39 E. Apra, M. Causa, M. Prencipe, R. Dovesi and V. R. Saunders, J. Phys.: Condens. Matter, 1993, 5, 2969-2976.

40 R. Krishnan, J. S. Binkley, R. Seeger and J. A. Pople, J. Chem. Phys., 1980, 72, 650-654.

41 M. Frisch, G. W. Trucks, H. B. Schlegel, G. E. Scuseria, M. A. Robb, J. R. Cheeseman, G. Scalmani, V. Barone, B. Mennucci, G. A. Petersson, H. Nakatsuji, M. Caricato, X. Li, H. P. Hratchian, A. F. Izmaylov, J. Bloino, G. Zheng, J. L. Sonnenberg, M. Hada, M. Ehara, K. Toyota, R. Fukuda, J. Hasegawa, M. Ishida, T. Nakajima, Y. Honda, O. Kitao, H. Nakai, T. Vreven, J. A. Montgomery, Jr., J. E. Peralta, F. Ogliaro, M. Bearpark, J. J. Heyd, E. Brothers, K. N. Kudin, V. N. Staroverov, R. Kobayashi, J. Normand, K. Raghavachari, A. Rendell, J. C. Burant, S. S. Iyengar, J. Tomasi, M. Cossi, N. Rega, J. M. J. M. Millam, M. Klene, J. E. Knox, J. B. Cross, V. Bakken, C. Adamo, J. Jaramillo, R. Gomperts, R. E. Stratmann, O. Yazyev, A. J. Austin, R. Cammi, C. Pomelli,
J. W. Ochterski, R. L. Martin, K. Morokuma, V. G. Zakrzewski, G. A. Voth, P. Salvador, J. J. Dannenberg, S. Dapprich, A. D. Daniels, Ö. Farkas, J. B. Foresman, J. V. Ortiz, J. Cioslowski and D. J. Fox, Gaussian 09 Revision D.01, Gaussian Inc., Wallingford, CT, 2009.

42 T. Yanai, D. P. Tew and N. C. Handy, Chem. Phys. Lett., 2004, 393, 51-57.

43 M. J. Peach, P. Benfield, T. Helgaker and D. J. Tozer, J. Chem. Phys., 2008, 128, 044118.

44 V. A. Mozhayskiy and A. I. Krylov, ezSpectrum, http://iopen shell.usc.edu/downloads.

45 A. Paulheim, C. Marquardt, H. Aldahhak, E. Rauls, W. G. Schmidt and M. Sokolowski, J. Phys. Chem. C, 2016, 120, 11926-11937.

46 J. P. Toennies, A. F. Vilesov and K. B. Whaley, Phys. Today, 2001, 54, 31-37.

47 R. Fatema, D. Van Winkle, J. Skofronick, S. Safron and F. Flaherty, Phys. Rev. B: Condens. Matter Mater. Phys., 2008, 77, 024305.

48 M. S. Kushwaha, IL Nuovo Cimento B, 1980, 60, 187-200.

49 V. Shklover, PhD thesis, University of Würzburg, 2002.

50 R. Scholz and M. Schreiber, Chem. Phys., 2006, 325, 9-21.

51 F. S. Tautz and J. A. Schaefer, J. Appl. Phys., 1998, 84, 6636-6643. 